\title{
Biochemical Changes and Cardiovascular Function in Parkinson's Disease: Precautionary Notes
}

\section{Ramon Cacabelos*}

Professor and Chairman of Genomic Medicine, Institute of Medical Science and Genomic Medicine, EuroEspes Biomedical Research Center,15166-Bergondo, Corunna, Spain

Parkinson's disease (PD) is the second most important neurodegenerative disorder in the elderly population, after Alzheimer's disease. With a prevalence ranging from 35.8 per 100,000 to 12,500 per 100,000 and annual incidence estimates ranging from 1.5 per 100,000 to 346 per 100,000 in different countries [1-3], PD represents today a major age-related problem of health $[4,5]$. Meta-analysis of the world wide data indicates a rising prevalence of $\mathrm{PD}$ with age (from 41 per 100,000 at $40-49$ years to 1,903 per 100,000 at over age 80 ). PD also shows a characteristic distribution by geographic location (a prevalence of 1,601 per 100,000 in patients from North America, Europe and Australia, and a prevalence of 646 per 100,000 in Asian patients) [6]. $\mathrm{PD}$ is more prevalent in males $(1,729$ per $100,000,>65 \mathrm{yrs})$ than in females $(1,644$ per 100,000$)$, with a peak prevalence in the age group of $\geq 90$ years $(4,633$ cases per 100,000$)$, and a mean prevalence of 1,680 per 100,000 in people older than 65 years of age [7]. Prevalence and incidence Male/Female ratios increase by 0.05 and 0.14 , respectively, per 10 years of age. Incidence is similar in men and women under 50 years $(\mathrm{M} / \mathrm{F}$ ratio $<1.2)$, and over 1.6 times higher in men than women over 80 years [8].

PD-related neurodegeneration is likely to occur several decades before the onset of the motor symptoms (rigidity, bradykinesia, resting tremor, postural instability) [9], resulting from the confluence of genomic defects, epigenetic aberrations and diverse environmental risk factors (toxins, drugs, pesticides, brain microtrauma, focal cerebrovascular damage).

Different genes distributed across the human genome have been associated with PD, including GBA, ADH1C, TBP SCA17, HDL4, ATXN2, MAPT, SNCA, PARK1-22, LRRK2, PINK1, CHCHD2, UCHL1, APOE, and many others [10,11]. All these genes are under the influence of the epigenetic machinery (DNA methylation, chromatin remodeling, histone modifications, miRNAs) that regulates their expression in different tissues and may contribute to selective nigrostriatal dopaminergic neurodegeneration. PD neuropathology is characterized by a selective loss of dopaminergic neurons in the substantia nigra pars compacta, with widespread involvement of other brain structures and peripheral tissues [9].

PD exhibits multiple biochemical changes in body fluids and in brain tissues, including neurotransmitters, neurotrophic factors, Lewy body components, abnormal proteins encoded by mutant PDassociated genes, transition metals, calcium and calcium-binding proteins, inflammation factors, ROS and mitochondrial anomalies, proteasome dysfunction, conformational changes in proteins, abnormal protein removal and degradation, hormonal dysregulation, apoptosis and alterations in transduction pathways [12,13]. Recent data indicate that endocrine dyscrasia and the subsequent loss of cell cycle control represent pathogenic events in the development of ageand sex-related neurodegenerative diseases, including Alzheimer's disease, stroke and PD [13]. However, a major question is whether these changes are primarily associated with pathogenic mechanisms or are the consequence of chronic treatment with antiparkinsonian drugs in the case of PD.
The introduction of L-DOPA in the 1960s represented a breakthrough in the treatment of $\mathrm{PD}$, and it continues to be the most effective symptomatic therapy in Parkinsonian disorders [14]. Levodopa (L-DOPA) is the natural isomer of the amino acid D,Ldihydroxyphenylalanine which was isolated from the bean of Vicia faba in the early 1910 s by Torquato Torquati, and chemically defined by Markus Guggenheim in 1913. In 1938, Peter Holtz discovered the enzyme L-dopadecarboxylase, which converts L-DOPA into dopamine [15]. Dopamine is transformed into noradrenaline by dopamine- $\beta$-hydroxylase. Both catecholamines are important neurotransmitters involved in different higher activities of the central nervous system. In addition to dopamine precursors (L-DOPA), other symptomatic treatments for PD include dopamine agonists (amantadine, apomorphine, bromocriptine, cabergoline, lisuride, pergolide, pramipexole, ropinirole, rotigotine), monoamine oxidase (MAO) inhibitors (selegiline, rasagiline), and catecholO-methyltransferase (COMT) inhibitors (entacapone, tolcapone) [16]. The initial complication of long-term L-DOPA therapy is the "wearing-off" phenomenon $[17,18]$, together with motor fluctuations and dyskinesia, which develop during the use of both L-DOPA and dopamine agonists [14,19-21]. Polypharmacy with antidepressants, antipsychotics, urological drugs, analgesics, antihistaminics and cholinesterase inhibitors also contributes to severe complications associated with the anticholinergic burden in PD [22]. Furthermore, gastrointestinal complications (constipation, sialorrhea, dysphagia, difficulty in mastication, choking/aspiration) [23], cardiovascular problems [24], neuroendocrine changes and psychiatric disorders are frequent in Parkinsonian patients chronically treated with conventional antiparkinsonian drugs [16,23]. The onset of these complications is also influenced by the genomic background of the patients [11]; and the efficacy and safety of the drugs currently consumed by those who suffer a Parkinsonian disorder is highly dependent on their pharmacogenomic profile [25-29]. Genes involved in the pharmacogenetic network include pathogenic, mechanistic, metabolic, transporter and pleiotropic genes, and all these genes are also under the influence of potential epigenetic aberrations [30-32]. In recent years, novel evidence has demonstrated the impact of pharmacogenetics on the efficacy and safety of most antiparkinsonian drugs [16,26-29]. In the particular case of L-DOPA, the ANKK1, BDNF, LRRK2, and PARK2 genes are pathogenic genes potentially involved in its effects. The $C C K$, CCKAR, CCKBR, DRD1, DRD2, DRD3, DRD4, DRD5, GRIN2A,

*Corresponding author: Ramon Cacabelos, Professor and Chairman of Genomic Medicine Institute of Medical Science and Genomic Medicine, EuroEspes Biomedical Research Center,15166-Bergondo, Corunna, Spain, Tel: 34981780505; E-mail: rcacabelos@euroespes.com

Received July 22, 2016; Accepted July 23, 2016; Published July 25, 2016

Citation: Cacabelos R (2016) Biochemical Changes and Cardiovascular Function in Parkinson's Disease: Precautionary Notes. Clin Med Biochem 2: e102. doi:10.4172/2471-2663.1000e102

Copyright: (C) 2016 Cacabelos R. This is an open-access article distributed under the terms of the Creative Commons Attribution License, which permits unrestricted use, distribution, and reproduction in any medium, provided the original author and source are credited. 
GRIN2B, HCRT, HOMER1, LMO3, and OPRM1 genes are mechanistic genes whose products influence L-DOPA efficacy and safety. L-DOPA is a substrate of enzymes encoded by the COMT, CYP1A2, CYP2B6, CYP2C19, CYP2D6, CYP3A4, CYP3A5, DBH, DDC, G6PD, MAOB, TH, UGT1A1, and UGT1A9 genes responsible for its metabolism. SLC6A3 is the major transporter of L-DOPA; and ACE, ACHE and $A P O E$ are pleiotropic players in L-DOPA effects [16]. ADORA2A SNPs and HOMER1 variants may be associated with L-DOPA-induced dyskinesia and psychotic symptoms [33,34]. A haplotype integrating -141CIns/Del, rs2283265, rs1076560, C957T, TaqIA and rs2734849 polymorphisms at the DRD2/ANKK1 gene region might also be associated with L-DOPA-induced motor dysfunction [35]; and SLC6A3 is a genetic modifier of the treatment response to L-DOPA in PD [36].

In a search for novel therapeutic options for PD [37,38], we had the opportunity of evaluating basal biochemical conditions and cardiovascular function in PD patients without any treatment at the moment of their first diagnosis, and in PD patients chronically treated with antiparkinsonian drugs for more than one year. Both groups exhibited substantial biochemical and cardiovascular differences. In addition to changes in blood biochemical (i.e., urea, creatinine, phosphorous, alkaline phosphatase, folate, cyanocobalamin) and hematological parameters (neutrophils, basophils) [38], the most significant differences were found in plasma neurotransmitters and hormones. For instance, as expected, basal dopamine levels in untreated patients were below $20 \mathrm{pg} / \mathrm{mL}(11.22 \pm 0.29 \mathrm{pg} / \mathrm{mL})(\mathrm{mean} \pm$ $\mathrm{SE}$ ) whereas basal dopamine levels in treated patients ranged from 21 to $30,000 \mathrm{pg} / \mathrm{mL})(2,139.23 \pm 804.72 \mathrm{pg} / \mathrm{mL})(\mathrm{p}<0.001)$. Chronic treatment with antiparkinsonian drugs reduces the levels of plasma serotonin, $\mathrm{FSH}, \mathrm{LH}$ and estrogen, and tends to increase the levels of adrenaline, histamine, ACTH, cortisol, and testosterone in a non-significant mode. Changes in neurotransmitters and endocrine parameters are highly influenced by the genomic profile of each patient $[37,38]$.

When PD patients are stratified by their cardiovascular function, as assessed with EKG, 39.50\% show an abnormal EKG, $19.32 \%$ are borderline, and $41.18 \%$ exhibit a normal EKG. Significant differences have been found in plasma neurotransmitter levels (dopamine, noradrenaline, adrenaline, serotonin, histamine) and hormones (prolactin, cortisol, LH, estrogens). High levels of noradrenaline, dopamine, histamine and serotonin, and lower levels of prolactin, $\mathrm{LH}$, testosterone and estrogens are more frequent among patients with abnormal EKG. It appears that a persistent overload of catecholamines and, to a lesser extent, histamine might contribute to cardiovascular dysfunction in PD patients. Since these patients undergo long-term periods of treatment with different antiparkinsonian drugs, which chronically alter neurotransmitter levels and hormonal regulation, some reflections are pertinent: (i) it is likely that the chronic overstimulation of the dopaminergic system at central and peripheral levels may become deleterious for cardio-cerebrovascular function and hormonal regulation; (ii) since the central dopaminergic dysfunction precedes the clinical onset of the disease by several decades, it is urgent to identify predictive biomarkers to protect the population at risk of suffering PD; (iii) the progression of PD for the past 50 years, in terms of gradual increase in prevalence and incidence rates, indicates that environmental toxicity may contribute to accelerate selective dopaminergic neurodegeneration; therefore, a better scrutiny of environmental risk factors is necessary to implement preventive programs in susceptible persons; (iv) most post-mortem neurochemical studies might be contaminated by chronic polypharmacy; (v) novel drugs and bio products for the treatment of PD should address dopaminergic neuroprotection to reduce premature neurodegeneration rather than dopaminergic overstimulation; and (vi) since biochemical changes and therapeutic outcomes are highly dependent upon the genomic profiles of PD patients, personalized treatments should rely on pharmacogenetic procedures to optimize therapeutics [16].

\section{References}

1. von Campenhausen S, Bornschein B, Wick R, Bötzel K, Sampaio C, et al (2005) Prevalence and incidence of Parkinson's disease in Europe. Eur Neuropsychopharmacol 15: 473-490.

2. Zou YM, Liu J, Tian ZY, Lu D, Zhou YY (2015) Systematic review of the prevalence and incidence of Parkinson's disease in the People's Republic of China. Neuropsychiatr Dis Treat 11: 1467-1472.

3. Muangpaisan W, Hori $\mathrm{H}$, Brayne $\mathrm{C}$ (2009) Systematic review of the prevalence and incidence of Parkinson's disease in Asia. J Epidemiol 19: 281-293.

4. Hirsch L, Jette N, Frolkis A, Steeves T, Pringsheim T (2016) The Incidence of Parkinson's Disease: A Systematic Review and Meta-Analysis. Neuroepidemiology 46: 292-300.

5. Savica R, Grossardt BR, Bower JH, Ahlskog JE, Rocca WA (2016) Time Trends in the Incidence of Parkinson Disease. JAMA Neurol.

6. Pringsheim T, Jette N, Frolkis A, Steeves TD (2014) The prevalence of Parkinson's disease: a systematic review and meta-analysis. Mov Disord 29: 1583-1590.

7. Riedel O, Bitters D, Amann U, Garbe E, Langner I (2016) Estimating the prevalence of Parkinson's disease (PD) and proportions of patients with associated dementia and depression among the older adults based on secondary claims data. Int J Geriatr Psychiatry.

8. Moisan F, Kab S, Mohamed F, Canonico M, Le Guern M, et al. (2015) Parkinson disease male-to-female ratios increase with age: French nationwide study and meta-analysis. J Neurol Neurosurg Psychiatry.

9. Miller DB, O'Callaghan JP (2015) Biomarkers of Parkinson's disease: Present and future. Metabolism 64: S40-S46.

10. Hernandez DG, Reed X, Singleton AB (2016) Genetics in Parkinson's disease Mendelian versus non-Mendelian inheritance. J Neurochem.

11. Hill-Burns EM, Ross OA, Wissemann WT, Soto-Ortolaza Al, et al. (2016) Identification of genetic modifiers of age-at-onset for familial Parkinson's disease. Hum Mol Genet.

12. Toulorge D, Schapira AH, Hajj R (2016) Molecular changes in the postmortem parkinsonian brain. J Neurochem.

13. Atwood CS, Bowen RL (2016) The endocrine dyscrasia that accompanies menopause and andropause induces aberrant cell cycle signaling that triggers re-entry of post-mitotic neurons into the cell cycle, neurodysfunction neurodegeneration and cognitive disease. Horm Behav 76: 63-80.

14. Katzenschlager R, Lees AJ (2002) Treatment of Parkinson's disease: levodopa as the first choice. J Neurol. 249: 19-24.

15. Oleh Hornykiewicz O (2010) A brief history of levodopa. J Neurol 257 (Supp 2): S249-S252.

16. Cacabelos R (2012) World Guide for Drug Use and Pharmacogenomics EuroEspes Publishing, Corunna.

17. Pahwa R, Lyons KE (2009) Levodopa-related wearing-off in Parkinson's disease: identification and management. Curr Med Res Opin. 25: 841-849.

18. Bhidayasiri R, Hattori N, Jeon B, Chen RS, Lee MK, et al. (2015) Asian perspectives on the recognition and management of levodopa 'wearing-off' in Parkinson's disease. Expert Rev Neurother 15: 1285-1297.

19. Haaxma CA, Horstink MW, Zijlmans JC, Lemmens WA, Bloem BR, et al (20015) Risk of Disabling Response Fluctuations and Dyskinesias for Dopamine Agonists Versus Levodopa in Parkinson's Disease. J Parkinsons Dis 5: 847-853.

20. Rascol O, Perez-Lloret S, Ferreira JJ (2015) New treatments for levodopainduced motor complications. Mov Disord 30: 1451-1460.

21. Stowe R, Ives N, Clarke CE, Deane K, van Hilten, et al. (2010) Evaluation of the efficacy and safety of adjuvant treatment to levodopa therapy in Parkinson $\mathrm{s}$ disease patients with motor complications. Cochrane Database Syst Rev 7 : CD007166. 
Citation: Cacabelos R (2016) Biochemical Changes and Cardiovascular Function in Parkinson's Disease: Precautionary Notes. Clin Med Biochem 2: e102. doi:10.4172/2471-2663.1000e102

22. Lertxundi U, Isla A, Solinis MA, Domingo-Echaburu S, Hernandez R, et al. (2015) Anticholinergic burden in Parkinson's disease inpatients. Eur J Clin Pharmacol. 71: 1271-1277.

23. Owolabi LF, Samaila AA, Sunmonu T (2014) Gastrointestinal complications in newly diagnosed Parkinson's disease: A case-control study. Trop Gastroenterol 35: $227-231$

24. Tran T, Brophy JM, Suissa S, Renoux C (2015) Risks of Cardiac Valve Regurgitation and Heart Failure Associated with Ergot- and Non-Ergot-Derived Dopamine Agonist Use in Patients with Parkinson's Disease: A Systematic Review of Observational Studies. CNS Drugs 29: 985-998.

25. Cacabelos R, Torrellas C, Cacabelos P, Villanueva MJ, Piñeiro $S$, et al. (2015) Pharmacogenetics of neurodegenerative disorders. In: Preventive and Predictive Genetics: Towards Personalized Medicine. Springer, pp: 173-240.

26. Altmann V, Schumacher-Schuh AF, Rieck M, Callegari-Jacques SM, Rieder $\mathrm{CR}$, et al. (2016) Influence of genetic, biological and pharmacological factors on levodopa dose in Parkinson's disease. Pharmacogenomics 17: 481-488.

27. Jiménez-Jiménez FJ, Alonso-Navarro H, García-Martín E, Agúndez JA (2016) Advances in understanding genomic markers and pharmacogenetics of Parkinson's disease. Expert Opin Drug Metab Toxicol 12: 433-448.

28. Kurzawski M, Białecka M, Droździk M (2015) Pharmacogenetic considerations in the treatment of Parkinson's disease. Neurodegener Dis Manag 5: 27-35.

29. Schumacher-Schuh AF, Rieder CR, Hutz MH (2014) Parkinson's disease pharmacogenomics: new findings and perspectives. Pharmacogenomics 15: 1253-1271.

30. Cacabelos R, Cacabelos P, Torrellas C, Tellado I, Carril JC (2014) Pharmacogenomics of Alzheimer's disease: novel therapeutic strategies for drug development. Methods Mol Biol 1175: 323-556.
31. Cacabelos $R$ (2014)) Epigenomic networking in drug development: from pathogenic mechanisms to pharmacogenomics. Drug Dev Res. 75: 348-365.

32. Cacabelos R, Torrellas C (2015) Epigenetics of aging and Alzheimer's disease: Implications for pharmacogenomics and drug response. International Journal of Molecular Sciences 16: 30483-30543.

33. Rieck M, Schumacher-Schuh AF, Callegari-Jacques SM, Altmann V, Schneider Medeiros $M$, et al. (2015) Is there a role for ADORA2A polymorphisms in levodopa-induced dyskinesia in Parkinson's disease patients? Pharmacogenomics 16: 573-582.

34. Schumacher-Schuh AF, Altmann V, Rieck M, Tovo-Rodrigues L, Monte TL, et al. (2014) Association of common genetic variants of HOMER1 gene with levodopa adverse effects in Parkinson's disease patients. Pharmacogenomics J 14: 289-294.

35. Rieck M, Schumacher-Schuh AF, Altmann V, Francisconi CL, Fagundes PT, et al. (2012) DRD2 haplotype is associated with dyskinesia induced by levodopa therapy in Parkinson's disease patients. Pharmacogenomics. 13: 1701-1710.

36. Moreau C, Meguig S, Corvol JC, Labreuche J, Vasseur F, et al. (2015) Polymorphism of the dopamine transporter type 1 gene modifies the treatment response in Parkinson's disease. Brain. 138: 1271-1283.

37. Cacabelos R (2016) Bioactive extract obtained from Vicia faba and its use in the treatment and/or prevention of neurodegenerative diseases. European Patent EP16382138.

38. Cacabelos R, Fernández-Novoa L, Alejo R, Corzo L, Alcaraz M, et al. (2016) E-PodoFavalin-15999 (Atremorine $\AA$ )-induced dopamine response in Parkinson's disease: Pharmacogenetics-related effects. J Genomic Med Pharmacogenomics, p: 1.
Citation: Cacabelos R (2016) Biochemical Changes and Cardiovascular Function in Parkinson's Disease: Precautionary Notes. Clin Med Biochem 2: e102. doi:10.4172/2471-2663.1000e102 